ISSN 0103-5150

Fisioter. Mov., Curitiba, v. 24, n. 1, p. 65-73, jan./mar. 2011

Licenciado sob uma Licença Creative Commons

\title{
Repercussões da fisioterapia na unidade de terapia intensiva neonatal
}

\author{
Repercussion of physiotherapy in the neonatal intensive \\ care unit
}

\section{Gabriela Arruda Reinaux de Vasconcelos ${ }^{[a]}$, Rita de Cássia Albuquerque Almeida ${ }^{[b]}$, Andrezza de Lemos Bezerra ${ }^{[c]}$}

[a] Fisioterapeuta pela Universidade Católica de Pernambuco (Unicap), Recife, PE - Brasil, e-mail: garv.fisio@gmail.com

[b] Fisioterapeuta, Especialista em Fisioterapia Pediátrica e Neonatal, Hospital Agamenon Magalhães e Faculdade Pernambucana de Saúde, Recife, PE - Brasil, e-mail: ritacassia@gmail.com

[c] Fisioterapeuta, Mestre, Professora da Escola Pernambucana de Saúde, Hospital Agamenon Magalhães e Faculdade Pernambucana de Saúde, Recife, PE - Brasil, e-mail: alemos4@gmail.com

\section{Resumo}

Objetivos: 0 objetivo deste trabalho é avaliar o impacto e os benefícios da intervenção do fisioterapeuta no desfecho dos recém-nascidos internados na unidade de terapia intensiva neonatal (UTIN) do Hospital Agamenon Magalhães (HAM), da cidade de Recife, PE, por comparação entre os anos de 2005 e 2007, em que houve aumento do tempo de permanência do fisioterapeuta na UTIN. Metodologia: A pesquisa foi realizada por análise de prontuários no sistema de arquivos médicos e estatísticos (Same) para adquirir informações sobre os recémnascidos (RNs) internados na UTIN no ano de 2005 e 2007. Em um total foram analisados 195 prontuários, sendo destes 110 do ano de 2005 e 85 de 2007. Os dados foram analisados por meio do programa SPSS versão 13.0 para Windows e Excel 2003. Resultados: Os dados obtidos demonstram que, no ano de 2007, os RNs tinham idade gestacional e peso, ao nascimento, menores quando comparados com o ano de 2005. Não houve diferença significativa, entre os anos de 2005 e 2007, quanto ao tempo de ventilação mecânica, tempo de ventilação não invasiva e tempo de oxigenoterapia, como era de se esperar, já que os RNs de 2007 eram menores. Foi observada, no entanto, uma diminuição significativa na fração inspirada de oxigênio no ano de 2007. Conclusão: De acordo com os resultados obtidos, observa-se uma influência positiva da maior permanência do fisioterapeuta na UTIN.

Palavras-chave: Unidade de terapia intensiva neonatal. Recém-nascidos prematuros. Fisioterapia. 
Abstract

Objectives: The aim of this study is to evaluate the impact and benefits of the physiotherapist intervention on the outcome of infants interned in the neonatal intensive care unit (Nicu) of Hospital Agamenon Magalhães (HAM), Recife, PE, comparing the years of 2005 and 2007, in which there was an increase of the physiotherapist's permanence at the Nicu. Methods: Data were collected from medical record of the years 2005 and 2007, accessed at the system of medical and statistical archives (Same). A total of 195 records were found and assessed, 110 of 2005 and 85 of 2007. Statistical analysis was processed by SPSS version 13.0 for Windows and Excel 2003. Results: The obtained data demonstrate that, at 2007, infants had lower gestational age and birthweight when compared to 2005. There was no significant difference, between the years 2005 and 2007, in relation to time of mechanical ventilation, time of non-invasive ventilation and time of oxygentherapy, as it would be expected, since the newborns of 2007 were smaller. It was observed a significant decrease in the oxygen inspired fraction at 2007. Conclusion: According to obtained results, it is observed a positive influence of the physiotherapist's longer permanence at the Nicu.

Keywords: Neonatal intensive care unit. Preterm infants. Physiotherapy.

\section{Introdução}

O seguimento da criança e do recém-nascido de alto risco é uma especialidade hoje estabelecida na maioria dos países desenvolvidos (1). No Brasil, as iniciativas para o trabalho de fisioterapia nas unidades de terapia intensiva neonatal (UTINs) iniciaram-se na década de 80. No primeiro momento, após a criação das UTINs, a preocupação maior era a de melhorar a sobrevida dos recém-nascidos (RNs) sem aumentar o número de complicações (2). Partindo desse preceito, pode-se afirmar que a fisioterapia é uma especialidade que vem progredindo por meio do esforço de profissionais que têm como proposta um atendimento diferenciado para os RNs de alto risco (3).

O RN na UTIN pode tornar-se instável pela própria doença de base ou em função do tratamento que é imposto, como também pela utilização de medicações ou ainda pela ventilação mecânica (7). Esses fatores podem contribuir para que os RNs internados nessas unidades fiquem suscetíveis a adquirir infecções ou outras complicações (8-10), evoluindo com necessidade de acompanhamento da fisioterapia.

A fisioterapia é uma modalidade terapêutica relativamente recente dentro das UTINs e que está em expansão, especialmente nos grandes centros, sendo realizada por meio de diversas técnicas, com o objetivo de diminuir o trabalho respiratório, manter a patência de vias aéreas e melhorar a ventilação e a troca gasosa $(4,5)$. Segundo a portaria do Ministério da Saúde n. 3.432, em vigor desde 12/8/1998, as unidades de terapia intensiva (UTIs) de hospitais com nível terciário devem contar com assistência fisioterapêutica com turno de no mínimo 12 horas/ dia, por diminuir as complicações e o período de hospitalização, reduzindo, consequentemente, os custos hospitalares (6).

A carga horária da fisioterapia na UTIN do HAM, no período de 2004 a 2005, era regulamentada em regime de 6 horas/dia, 5 dias/semana, de atendimento fisioterapêutico, o qual era realizado em um único turno ficando os demais horários sem assistência da fisioterapia. No entanto, a partir de 2006 (período de transição entre os dois regimes) começou a ser implantado na UTIN do HAM o regime de 12 horas/dia, 7 dias/semana - sendo esse regime efetivado em 2007 -, contando com assistência da equipe de fisioterapia em turno integral.

Com o intuito de exercer a prática baseada em evidências, este estudo teve como objetivo avaliar o impacto e os benefícios da mudança do regime de trabalho da equipe de fisioterapia na UTIN do HAM, nos anos de 2005 e 2007, sobre o desfecho dos RNs em relação ao suporte ventilatório.

\section{Metodologia}

A pesquisa caracteriza-se como coorte retrospectiva, sendo realizada por análise de prontuários. A população abrangida constituiu-se de RNs internados na UTIN do HAM durante o período de janeiro a dezembro de 2005 e janeiro a dezembro de 2007, 
sendo excluídos aqueles que passaram apenas 24 horas na UTIN. 0 projeto de pesquisa foi submetido e aprovado pelo Comitê de Ética e Pesquisa do HAM, com registro no CEP n. 200/2008.

Solicitou-se à diretoria do sistema de arquivos médicos e estatísticos (Same) e Clínica Médica do HAM uma permissão para coletar os dados nos prontuários dos RNs, sendo a pesquisa realizada no próprio Same. Apenas foram registradas anotações referentes ao formulário da ficha de avaliação, mantendo sigilo sobre qualquer informação retirada.

Para verificar as informações foi utilizada uma ficha de coleta de dados, para registro das variáveis a serem estudadas, elaborada pelas pesquisadoras, onde era discriminado o código do prontuário do RN e nome da mãe, para identificação e pesquisa destes no Same. Esses registros foram coletados anteriormente no livro de admissão da UTIN. A ficha de avaliação continha, na anamnese: dados da mãe, dados do parto e escala de avaliação de risco de mortalidade neonatal. Em relação ao suporte ventilatório: dados dos modos ventilatórios utilizados assistência ventilatória mecânica invasiva (AVM), ventilação não invasiva (VNI) e pressão positiva contínua nas vias aéreas (CPAP). Também foi notificada a realização do atendimento fisioterapêutico no RN durante o período de internação.

Para os estudos estatísticos foram utilizados os softwares SPSS 13.0 para Windows e Excel 2003. Para as variáveis quantitativas foi realizado o teste de normalidade de Kolmogorov-Smirnov. Na comparação entre variáveis quantitativas foram utilizados o teste $\mathrm{t}$ de Student, apresentando os resultados por meio de média e desvio-padrão, e teste MannWhitney, apresentando os resultados por meio de mediana e quartis. Para verificar a existência de associação entre as variáveis categóricas, o teste exato de Fisher e o teste Qui-quadrado. Os dados estão apresentados em tabelas com suas respectivas frequências absolutas e relativas. Todos os testes foram aplicados com $95 \%$ de confiança.

\section{Resultados}

A lista inicial de RNs contava com 368 registros. Não foi possível resgatar todos os prontuários no Same, durante a coleta de dados, havendo perda de 100 prontuários: 75 do ano de 2005 e 25 do ano de 2007. A amostra foi composta por 268 prontuários, dos quais 73 (35 no ano de 2005 e 38 no ano de 2007) foram encaixados no critério de exclusão, totalizando uma amostra de 195 prontuários: 110 referentes aos RNs que foram admitidos no HAM e internados na UTIN durante o período de janeiro a dezembro de 2005 e 85 durante o período de janeiro a dezembro de 2007.

A Tabela 1 demonstra os dados dos RNs internados na UTIN, nos anos de 2005 e 2007, relacionados às condições maternas. Observou-se homogeneidade, entre os dois grupos, em todos os cruzamentos da Tabela 1 em relação: à idade da mãe, ao tipo de parto, ao pré-natal, ao tipo de gestação e a intercorrências durante a gestação.

Tabela 1 - Dados maternos dos recém-nascidos internados na unidade de terapia intensiva neonatal do Hospital Agamenon Magalhães em Recife, PE, no período de janeiro a dezembro de 2005 e 2007

\begin{tabular}{|c|c|c|c|c|c|}
\hline \multirow[b]{3}{*}{ Variáveis } & \multicolumn{4}{|c|}{ Ano } & \multirow[b]{3}{*}{$\mathrm{p}$-valor } \\
\hline & \multicolumn{2}{|c|}{2005} & \multicolumn{2}{|c|}{2007} & \\
\hline & $n$ & $\%$ & $n$ & $\%$ & \\
\hline \multicolumn{6}{|c|}{ Idade da mãe } \\
\hline$<20$ & 23 & 20,9 & 17 & 20,0 & 0,238 * \\
\hline $20 \mid-30$ & 54 & 49,1 & 33 & 38,8 & \\
\hline$\geq 30$ & 33 & 30,0 & 35 & 41,1 & \\
\hline \multicolumn{6}{|c|}{ Tipo de gestação } \\
\hline Única & 99 & 90,0 & 79 & 92,9 & 0,641 * \\
\hline Múltipla & 11 & 10,0 & 6 & 7,1 & \\
\hline
\end{tabular}


Tabela 1 - Dados maternos dos recém-nascidos internados na unidade de terapia intensiva neonatal do Hospital Agamenon Magalhães em Recife, PE, no período de janeiro a dezembro de 2005 e 2007

(Conclusão)

\begin{tabular}{|c|c|c|c|c|c|}
\hline \multirow[b]{3}{*}{ Variáveis } & \multicolumn{4}{|c|}{ Ano } & \multirow[b]{3}{*}{$\mathrm{p}$-valor } \\
\hline & \multicolumn{2}{|c|}{2005} & \multicolumn{2}{|c|}{2007} & \\
\hline & $\mathrm{n}$ & $\%$ & $\mathrm{n}$ & $\%$ & \\
\hline \multicolumn{6}{|l|}{ Pré-natal } \\
\hline $\operatorname{Sim}$ & 105 & 95,5 & 83 & 97,6 & 0,472 ** \\
\hline Não & 5 & 4,5 & 2 & 2,4 & \\
\hline \multicolumn{6}{|c|}{ Tipo de parto } \\
\hline Pélvico & 53 & 48,2 & 42 & 49,4 & 0,979 * \\
\hline Cesário & 57 & 51,8 & 43 & 50,6 & \\
\hline \multicolumn{6}{|l|}{ HAS } \\
\hline $\operatorname{Sim}$ & 17 & 15,5 & 13 & 15,3 & \\
\hline Não & 93 & 84,5 & 72 & 84,7 & 1,000 * \\
\hline \multicolumn{6}{|c|}{ Pré-eclâmpsia } \\
\hline Sim & 7 & 6,4 & 6 & 7,1 & 1,000 * \\
\hline Não & 103 & 93,6 & 79 & 92,9 & \\
\hline \multicolumn{6}{|c|}{ Diabetes mellitus } \\
\hline Sim & 14 & 12,7 & 6 & 7,1 & 0,291 * \\
\hline Não & 96 & 87,3 & 79 & 92,9 & \\
\hline \multicolumn{6}{|c|}{ Diabetes gestacional } \\
\hline Sim & 4 & 3,6 & 5 & 5,9 & 0,507 ** \\
\hline Não & 106 & 96,4 & 80 & 94,1 & \\
\hline \multicolumn{6}{|c|}{ Infecção do trato urinário } \\
\hline $\operatorname{Sim}$ & 48 & 43,6 & 33 & 38,8 & 0,596 * \\
\hline Não & 62 & 56,4 & 52 & 61,2 & \\
\hline \multicolumn{6}{|l|}{ Sífilis } \\
\hline Sim & 3 & 2,7 & 3 & 3,5 & $1,000 * *$ \\
\hline Não & 107 & 97,3 & 82 & 96,5 & \\
\hline \multicolumn{6}{|c|}{ Soropositivo (AIDS) } \\
\hline Sim & 1 & 0,9 & 3 & 3,5 & 0,320 ** \\
\hline Não & 109 & 99,1 & 82 & 96,5 & \\
\hline
\end{tabular}

Legenda: * = teste Qui-quadrado; ** = teste exato de Fisher; HAS = hipertensão arterial sistêmica; PE = pré-eclâmpsia; DM = diabetes mellitus; $\mathrm{DG}=$ diabetes gestacional; ITU = infecção do trato urinário.

A Tabela 2 demonstra os dados dos RNs ao nascimento, em que é observada diferença estatisticamente significante em relação à idade gestacional e ao peso ao nascimento, os quais foram menores em
2007. Também se pode notar que em 2007 houve maior necessidade de reanimação ao nascimento. Tal necessidade foi estatisticamente significativa quando comparada ao ano de 2005. 
Tabela 2 - Características dos recém-nascidos internados na unidade de terapia intensiva neonatal do Hospital Agamenon Magalhães em Recife, PE, no período de janeiro a dezembro de 2005 e 2007

\begin{tabular}{|c|c|c|c|c|c|}
\hline \multirow[b]{3}{*}{ Variáveis } & \multicolumn{4}{|c|}{ Ano } & \multirow[b]{3}{*}{$\mathrm{p}$-valor } \\
\hline & \multicolumn{2}{|c|}{2005} & \multicolumn{2}{|c|}{2007} & \\
\hline & n & $\%$ & n & $\%$ & \\
\hline \multicolumn{6}{|l|}{ Sexo } \\
\hline Feminino & 60 & 54,5 & 45 & 52,9 & 0,938 * \\
\hline Masculino & 50 & 45,5 & 40 & 47,1 & \\
\hline \multicolumn{6}{|c|}{ Peso ao nascimento } \\
\hline$<1000$ & 5 & 4,5 & 14 & 16,5 & $0,003 * *$ \\
\hline $1000 \mid-1500$ & 32 & 29,1 & 18 & 21,2 & \\
\hline $1500 \mid-2500$ & 45 & 41,0 & 45 & 52,9 & \\
\hline $2500 \mid-3000$ & 21 & 19,1 & 7 & 8,2 & \\
\hline $3000 \mid-4000$ & 5 & 4,5 & 1 & 1,2 & \\
\hline$\geq 4000$ & 2 & 1,8 & 0 & 0,0 & \\
\hline \multicolumn{6}{|c|}{ Necessidade de reanimação ao nascimento } \\
\hline Sim & 46 & 41,8 & 56 & 65,9 & 0,001 * \\
\hline Não & 64 & 58,2 & 29 & 34,1 & \\
\hline \multicolumn{6}{|c|}{ Síndrome do desconforto respiratório } \\
\hline Sim & 67 & 60,9 & 63 & 74,1 & 0,074 * \\
\hline Não & 43 & 39,1 & 22 & 25,9 & \\
\hline \multicolumn{6}{|c|}{ Taquipneia transitória do recém-nascido } \\
\hline $\operatorname{Sim}$ & 34 & 30,9 & 15 & 17,6 & 0,051 * \\
\hline Não & 76 & 69,1 & 70 & 82,4 & \\
\hline \multicolumn{6}{|c|}{ Síndrome de aspiração do mecônio } \\
\hline $\operatorname{Sim}$ & 4 & 3,6 & 1 & 1,2 & $0,389 * *$ \\
\hline Não & 106 & 96,4 & 84 & 98,8 & \\
\hline \multicolumn{6}{|c|}{ Cardiopatias congênitas } \\
\hline $\operatorname{Sim}$ & 3 & 2,7 & 0 & 0,0 & $0,259 * *$ \\
\hline \multirow[t]{2}{*}{ Não } & 107 & 97,3 & 85 & 100,0 & \\
\hline & Mediana & Q1 ; Q3 & Mediana & Q1；Q3 & \\
\hline Idade gestacional & 33,1 & 28,$4 ; 34,6$ & 31,0 & 26,$0 ; 33,9$ & $0,013 * * *$ \\
\hline
\end{tabular}

Legenda: ${ }^{*}=$ teste Qui-quadrado; ${ }^{* *}=$ teste exato de Fisher; ${ }^{* \star *}=$ teste Mann-Whitney.

Em relação às patologias respiratórias, observase que no ano de 2005 houve incidência maior de taquipneia transitória do recém-nascido (TTRN), e que no ano de 2007 houve uma incidência maior, embora sem diferença estatisticamente significativa, de síndrome do desconforto respiratório (SDR).

No estudo, não foi possível comparar os valores da escala de mortalidade neonatal (CRIB) entre os 
anos de 2005 e 2007, pois houve perda de mais de $10 \%$ do total da amostra durante a coleta de dados.

Na Tabela 3, estão demonstrados os dados relacionados a dias de internação e de suporte ventilatório dos RNs internados na UTIN do HAM, no período de 2005 e 2007, em que se verifica que a mediana dos dias de internação e do tempo de utilização de cada suporte ventilatório (AVM, VNI e CPAP) é similar, não ocorrendo aumento desse tempo no ano de 2007, como era de se esperar pela IG e peso dos RNs desse ano. Em relação ao impacto da mudança do regime de trabalho de 6 horas/dia, no ano de 2005, para 12 horas/dia, no ano de 2007, nesses parâmetros, foi verificado que só houve diferença estatisticamente significante em todas as frações inspiradas de oxigênio $\left(\mathrm{FiO}_{2}\right)$, que foram menores em 2007 se comparadas ao ano de 2005.

Em 2005 houve maior utilização de modos ventilatórios não invasivos (VNI e CPAP), visto que os RNs tinham patologias respiratórias mais leves, portanto mais estáveis do ponto de vista respiratório, que os RNs do ano de 2007, em que houve maior uso de assistência ventilatória mecânica invasiva (AVM).

$\mathrm{Na}$ Tabela 4, evidencia-se o aumento significativo na quantidade de atendimentos realizados na
UTIN do HAM, durante o ano de 2007, em relação ao ano de 2005.

\section{Discussão}

Com o surgimento e a implementação de ambientes para cuidados neonatais, a assistência perinatal avança em parceria com as inovações tecnológicas que beneficiam o diagnóstico precoce e, consequentemente, o tratamento do RN (11). No entanto, ainda é considerado elevado o número de internações dos RNs nas UTINs, em decorrência dos diagnósticos da prematuridade, muito baixo peso ao nascer, anóxia perinatal, malformações, dentre outros, que os predispõem a tratamentos especializados para sobreviver (12).

Outros estudos demonstram que os RNs que apresentam alguns desses fatores neonatais, como peso de nascimento menor a $1.500 \mathrm{~g}$, RN pré-termo, RN pequeno para a idade gestacional e RN com malformações congênitas, estão mais suscetíveis a ter hipóxia ao nascer $(13,14)$. Esses dados corroboram o achado deste estudo, em que houve maior necessidade de reanimação ao nascimento - na sala de parto -,

Tabela 3 - Dias de internação e suporte ventilatório dos recém-nascidos internados na UTIN do HAM em Recife, PE, no período de janeiro a dezembro de 2005 e 2007

\begin{tabular}{|c|c|c|c|c|c|}
\hline \multirow[b]{3}{*}{ Variáveis } & \multicolumn{4}{|c|}{ Ano } & \multirow[b]{3}{*}{$\mathrm{p}$-valor } \\
\hline & \multicolumn{2}{|c|}{2005} & \multicolumn{2}{|c|}{2007} & \\
\hline & Mediana & Q1 ; Q3 & Mediana & Q1 ; Q3 & \\
\hline Dias de internação & 7,0 & 3,$0 ; 18,0$ & 4,0 & 3,$0 ; 14,0$ & 0,129 * \\
\hline Tempo de assistência ventilatória mecânica (horas) & 72,0 & 34,$5 ; 103,0$ & 72,0 & 48,$0 ; 96,0$ & 0,722 * \\
\hline Tempo de CPAP (horas) & 18,0 & 12,$0 ; 48,0$ & 18,0 & 12,$0 ; 30,0$ & 0,394 * \\
\hline Tempo de pronga (VNI + CPAP) (horas) & 22,0 & 13,$0 ; 62,5$ & 24,0 & 15,$0 ; 47,5$ & 0,794 * \\
\hline Tempo total de oxigênio (horas) & 67,0 & 40,$8 ; 138,0$ & 66,0 & 41,$0 ; 120,0$ & 0,869 * \\
\hline Fração inspirada de oxigênio AVM (\%) & 60,0 & 50,$0 ; 90,0$ & 60,0 & 40,$0 ; 70,0$ & 0,017 * \\
\hline Fração inspirada de oxigênio VNI (\%) & 40,0 & 40,$0 ; 40,0$ & 30,0 & 30,$0 ; 40,0$ & 0,001 * \\
\hline Fração inspirada de oxigênio CPAP (\%) & 40,0 & 30,$0 ; 40,0$ & 30,0 & 30,$0 ; 35,0$ & $<0,001$ * \\
\hline \multirow[t]{2}{*}{ Fração inspirada de oxigênio Halo (\%) } & 40,0 & 30,$0 ; 40,0$ & 35,0 & 30,$0 ; 40,0$ & 0,043 * \\
\hline & \multicolumn{2}{|c|}{ Média \pm DP } & \multicolumn{2}{|c|}{ Média \pm DP } & \\
\hline Tempo de ventilação não invasiva (horas) & \multicolumn{2}{|c|}{$32,8 \pm 24,60$} & \multicolumn{2}{|c|}{$34,5 \pm 17,34$} & 0,796 ** \\
\hline
\end{tabular}

Legenda: * = teste Mann-Whitney; ** teste t de Student. 
Tabela 4 - Atendimentos de fisioterapia realizados nos RNS internados na UTIN do HAM em Recife, PE, no período de janeiro a dezembro de 2005 e 2007

\begin{tabular}{|c|c|c|c|c|c|}
\hline \multirow[b]{3}{*}{ Variáveis } & \multicolumn{4}{|c|}{ Ano } & \multirow[b]{3}{*}{$\mathrm{p}$-valor } \\
\hline & \multicolumn{2}{|c|}{2005} & \multicolumn{2}{|c|}{2007} & \\
\hline & $\mathrm{n}$ & $\%$ & $\mathrm{n}$ & $\%$ & \\
\hline \multicolumn{6}{|c|}{ Fisioterapia } \\
\hline Sim & 68 & 61,8 & 80 & 94,1 & $<0,001^{\star}$ \\
\hline Não & 42 & 38,2 & 5 & 5,9 & \\
\hline
\end{tabular}

Legenda: * $=$ teste Qui-quadrado.

no ano de 2007, visto que os RNs desse ano estavam mais sujeitos a ter hipóxia ao nascimento porque eram mais prematuros e de extremo baixo peso.

A relação de baixo peso dos RNs, encontrados no ano de 2007, denotam alto risco para apresentar morbidades e longo período de internação $(15,16)$. 0 peso inferior a $1.000 \mathrm{~g}$ é denominado "extremo baixo peso de nascimento" e pode decorrer de uma interrupção precoce da gestação e/ou por retardo no crescimento intrauterino (17).

0 peso ao nascer pode ser considerado, isoladamente, um dos principais fatores correlacionados à morbidade e à mortalidade neonatal, portanto, constitui um preditor de saúde imediato e futuro do RN (18-20). Dados de um estudo semelhante, realizado em uma maternidade privada de São Paulo, mostraram que $69,1 \%$ dos bebês nasceram por cesárea, tiveram peso ao nascer e idade gestacional, respectivamente, de 1.170 g e 31 semanas (21). Dados semelhantes foram encontrados neste estudo para os RNs do ano de 2007.

Num estudo realizado em um hospital privado de São Paulo, o tempo médio de internação hospitalar foi de 49,5 dias (21). Esses resultados mostraram que, para garantir a sobrevida dessas crianças, há necessidade de longo período de cuidado direto em ambiente hospitalar, e de procedimentos e terapêuticas complexas para recuperar a saúde e, sobretudo, prevenir as complicações advindas do tratamento intensivo e da internação prolongada (21). Nosso estudo limitou o acompanhamento à permanência do RN na UTIN, observando-se que, no ano de 2007, o tempo de internamento foi similar ao do ano de 2005, ou seja, não aumentou como era esperado, já que os RNs de 2007 tinham IG e peso menores.
Neste estudo, observou-se a manutenção do valor de fração inspirada de oxigênio $\left(\mathrm{FiO}_{2}\right)$, em níveis mais baixos em todas as variáveis estudadas (AVM, VNI, CPAP e Halo), no ano de 2007, o que minimiza os riscos de toxicidade do oxigênio (22).

A maior utilização de AVM nos RNs no ano de 2007 pode ser explicada pelo fato destes, em particular os prematuros, não possuírem estrutura e função pulmonar adequadas (23). Em algumas situações, o RN prematuro precisa de AVM para assegurar as trocas gasosas. Essa modalidade de suporte ventilatório ajuda a melhorar a ventilação alveolar, diminuindo o trabalho respiratório e reexpandindo as áreas atelectasiadas, contudo existem os riscos de seus efeitos adversos $(24,25)$. Entretanto, o tempo de permanência em AVM foi similar entre os anos de 2005 e 2007, situação diferente da esperada, em que RNs mais imaturos necessitariam de um tempo maior em suporte ventilatório invasivo.

No ano de 2005 foi evidenciado o uso maior de VNI. A patologia respiratória de maior incidência nesse ano foi a TTRN, que tem menos comprometimento da função pulmonar e é de resolução mais rápida. Nesse contexto, a VNI tem como objetivos melhorar a fadiga muscular e a capacidade residual funcional, por meio da diminuição de áreas de atelectasias, e melhorar, também, a troca gasosa $(26,27)$.

Apesar da menor quantidade de atendimentos da fisioterapia nos RNs em 2005, há estudos em que se denota que a fisioterapia respiratória está cada vez mais integrada nos serviços de UTIN, pois é uma forma especial de atendimento (28), como foi visto no ano de 2007 com o aumento significativo da atuação do fisioterapeuta. A prática fisioterapêutica é parte da assistência multidisciplinar aos RNs pré-termo 
(RNPT) sob cuidados intensivos e tem como objetivo prevenir e minimizar as complicações respiratórias decorrentes da própria prematuridade e da AVM e otimizar a função pulmonar de modo a facilitar as trocas gasosas e, assim, promover uma evolução clínica favorável $(28,29)$.

As indicações de intervenção fisioterapêutica e os tipos de condutas utilizadas variam de acordo com o local e o preparo técnico do profissional (30). Existem locais em que a indicação da fisioterapia é determinada pelo médico e locais em que todos os pacientes internados na UTIN recebem atendimento de fisioterapia (30). Da mesma forma, é discutível se a atuação do profissional estaria relacionada com a precocidade de alta hospitalar, já que a atuação do fisioterapeuta poderia estar relacionada a um menor índice de complicações. Esse fato seria conveniente para o sistema de saúde por, consequentemente, reduzir despesas hospitalares e garantir rotatividade de leitos para pacientes mais graves (31).

\section{Conclusão}

Os resultados evidenciaram uma interferência positiva da mudança de regime dos profissionais de fisioterapia no HAM, com maior permanência do fisioterapeuta na UTIN no ano de 2007, tendo repercussões benéficas, em que se observou: tempo similar de internação, tempo similar de AVM, VNI e CPAP, comparando-se com o ano de 2005; menor $\mathrm{FiO}_{2}$ (em AVM, VNI, CPAP e Halo), comparando-se ao ano de 2005.

\section{Agradecimentos}

Aos funcionários do Same do Hospital Agamenon Magalhães que colaboraram com a procura e o resgate dos prontuários dos anos de 2005 e 2007.

\section{Referências}

1. Garcia JM, Nicolau CM. Assistência fisioterápica aos recém-nascidos do berçário anexo à Maternidade do Hospital das Clínicas da Faculdade de Medicina da Universidade de São Paulo. Rev Fisioter Univ São Paulo. 1996;3:38-46.
2. Krause MF, Hoehn TMD. Chest physiotherapy in mechanically ventilated children: a review. Crit Care Med. 2000;28(5):1648-51.

3. Machado CE, Jorge MSB. Ser profissional de saúde em uma unidade neonatal de alto e médio risco: o visível e o invisível. Estud Psicol Campinas. 2005;22(2): 197-204.

4. Haddad ER, da Costa LCD, Negrini F, Sampaio MM. Abordagens fisioterapêuticas para remoção de secreções das vias aéreas em recém-nascidos: relato de casos. Pediatria (São Paulo). 2006; 28(2):135-40.

5. Coppo MRC. Efeitos da técnica de aumento do fluxo expiratório sobre a função pulmonar em lactentes com insuficiência respiratória aguda em ventilação mecânica [dissertação]. Campinas: Unicamp; 2005.

6. Nicolau CM, Lahóz AL. Fisioterapia respiratória em terapia intensiva pediátrica e neonatal: uma revisão baseada em evidências. Pediatria. 2007;29(3): 216-21.

7. Stiller K. Physiotherapy in intensive care: towards an evidence-based practice. Chest. 2000;118(6):1801-13.

8. Lopes JMA. Apnéia neonatal. Jornal de Pediatria (Rio de Janeiro). 2001; 77(Supl.1): 97-103.

9. Serra SOA, Vieira MA, Barbeira CBS. Caracterização dos recém-nascidos internados na unidade de terapia intensiva neonatal de um hospital universitário. Rev Pediatria Atual. 2004;2:42-7.

10. Feltrim MIZ, Parreira VF. Fisioterapia respiratória. Consenso de Lyon (São Paulo). 2001;9-47.

11. Nicolau CM, Falcão MC. Efeitos da fisioterapia respiratória em recém-nascidos: análise crítica da literatura. Rev Paul Pediatria. 2007;25(1):72-5.

12. Ribeiro AP, Barros CB, Bettin DC, Piper EM, dos Santos GL, Fernandes GS, et al. Atuação da fisioterapia sobre o tempo de internação dos neonatos prétermo acometidos por distúrbios respiratórios na UTI neonatal do Hospital Universitário São Francisco de Paula. Rev de Saúde da UCPEL. 2007;1(1):54-9.

13. Salvo FH, Flores AJ, Alarcón RJ, Nachar HR, Paredes VA. Factores de riesgo de test de Apgar bajo en recién nacidos. Rev Chil Pediatr. 2007;78(3):253-60.

14. de Carvalho ABR, de Brito ASJ, Matsuo T. Assistência à saúde e mortalidade de recém-nascidos de muito baixo peso. Rev Saúde Pública. 2007;41(6):1003-12. 
15. Tronchin DMR, Tsunechiro MA. Prematuros de muito baixo peso: do nascimento ao primeiro ano de vida. Rev Gaúcha de Enferm. 2007;28(1):79-88.

16. Enes CC. Fatores de risco gestacionais para o peso do primogênito. Pediatria (São Paulo). 2007;29(3): 168-75.

17. Duarte JLMB, Mendonça GAS. Fatores associados à morte neonatal em recém-nascidos de muito baixo peso em quatro maternidades no município do Rio de Janeiro, Brasil. Cad Saúde Pública. 2005;21(1): 181-91.

18. Marino WT. Estudo descritivo dos recém-nascidos de muito baixo peso em uma maternidade de nível terciário [dissertação]. São Paulo: Faculdade de Saúde Pública, Universidade de São Paulo; 2001.

19. Prigenzi ML, Trindade CEP, Rugolo LMSS, Silveira LVA. Fatores de risco associados à mortalidade de recémnascidos de muito baixo peso na cidade de Botucatu, São Paulo, no período 1995-2000. Rev Bras Saude Mater Infant. 2008;8(1):93-101.

20. Carvalho PI, Pereira PMH, de Frias PG, Vidal SA, Figueiroa JN. Fatores de risco para a mortalidade neonatal em coorte hospitalar de nascidos vivos. Epidemiol Ser Saúde. 2007;16(3):185-94.

21. Tiago LF, Caldeira AP, Vieira MA. Fatores de risco de baixo peso ao nascimento em maternidade pública do interior de Minas Gerais. Pediatria (São Paulo). 2008;30(1):8-14.

22. II Consenso Brasileiro de Ventilação Mecânica. J Pneumol. 2000;26(Supl. 2):60-3.

23. Barbosa AL, Campos ACS, Chaves EMC. Complicações não clínicas da ventilação mecânica: ênfase no cuidado de enfermagem neonatal. Acta Paul Enferm. 2006;19(4):439-43.

24. Selestrin CC, de Oliveira AG, Ferreira C, de Siqueira AAF, de Abreu LC, Murad N. Avaliação dos parâmetros fisiológicos em recém nascidos pré-termo em ventilação mecânica após procedimentos de fisioterapia neonatal. Rev Bras Crescimento Desenvolv. Hum. 2007;17(1):146-55.
25. Nicolau CM. Repercussões da fisioterapia respiratória sobre a função cardiopulmonar em RNPT submetidos à ventilação mecânica [dissertação]. São Paulo: Universidade Federal de São Paulo; 2006.

26. Millar D, Kirpalani H. Benefits of noninvasive ventilation. Indian Pediatrics. 2004;41(10):1008-17.

27. Loh LE, Chan YH, Chan I. Noninvasive ventilation in children: a review. J Pediatr (Rio Janeiro). 2007;83(2): 91-99.

28. Nicolau CM, Pigo JDC, Bueno M, Falcão MC. Avaliação da dor em recém-nascidos prematuros durante a fisioterapia respiratória. Rev Bras Saude Mater Infant. 2008;8(3):285-90.

29. Abreu LC, Angheben JMM, Braz PF, de Oliveira AG, Falcão MC, Saldiva PHN. Effect of the neonatal physiotherapy in the heart rate in preterm infant with respiratory distress syndrome after replacement of exogenous surfactant. Arq Med ABC. 2006;31(1):5-11.

30. Falcão MC. Fisioterapia respiratória no período neonatal. Pediatria (São Paulo). 2006;28(3):211-2.

31. Juliani RCTP, Lahoz ALC, Nicolau CM, Paula LCS, Cunha MT. Fisioterapia nas unidades de terapia intensiva pediátrica e neonatal. Programa Nacional de Educação Continuada em Pediatria. PRONAP 2003/2004; 70:9-24.

Recebido: $18 / 05 / 2010$

Received: 05/18/2010

Aprovado: 27/07/2010

Approved: 07/27/2010 